\title{
Uma Investigação sobre os Fatores Críticos de Sucesso em Iniciativas de Melhoria de Processos de Software
}

\author{
Mariano Angel Montoni, Ana Regina Cavalcanti da Rocha \\ COPPE/UFRJ - Universidade Federal do Rio de Janeiro \\ Caixa Postal 68511 - CEP 21945-970 - Rio de Janeiro - RJ - Brasil \\ \{mmontoni, darocha\}@es.ufrj.br
}

\begin{abstract}
Resumo. As organizações enfrentam diversas dificuldades na condução de iniciativas de melhoria de processos de software. As causas dessas dificuldades estão relacionadas a aspectos de caráter sócio-cultural, tecnológico e organizacional. A compreensão dos fatores críticos de sucesso em iniciativas de melhoria de processos de software é fundamental para apoiar a gerência de iniciativas de melhoria e de melhores práticas de implementação. Este trabalho apresenta uma investigação conduzida por meio da aplicação de métodos qualitativos e quantitativos de análise de dados, visando construir um framework teórico que ajude a explicar o processo social do comportamento humano que rege a implementação de melhorias em processos de software. O framework teórico é constituído de conceitos e relacionamentos de influência, fundamentados em um conjunto de proposições (hipóteses), representando a visão e a perspectiva de implementadores de melhorias em processos de software.
\end{abstract}

\begin{abstract}
The organizations face a variety of difficulties in the conduction of software processes improvement initiatives. The causes of such difficulties are related to socio-cultural, technological and organizational aspects. The understanding of the critical success factors in software processes improvement initiatives is essential to support the management of improvement initiatives and implementation best practices. This work presents an investigation conducted by applying both qualitative and quantitative data analysis methods, aiming to construct a theoretical framework that helps to explain the social process of human behavior that governs software processes improvement implementation. The theoretical framework is constituted of concepts and influence relationships, grounded on a set of propositions (hypotheses), representing the vision and the perspective of software processes improvement practitioners.
\end{abstract}

\section{Introdução}

Para apoiar a implementação de melhorias em processos de software, diversos programas têm sido conduzidos com o propósito de desenvolver e aprimorar frameworks de melhores práticas de desenvolvimento de software, por exemplo, o CMMI (Sei, 2006) e o MPS.BR (Softex, 2009). No entanto, apesar de ter crescido de forma geral, nos últimos anos, a adoção de normas e modelos de referência para melhoria de processos, a quantidade de organizações que adotam esses modelos é uma parcela reduzida da população total de organizações de software (Staples et al., 2007). 
Estudos conduzidos para entender as razões do porquê as organizações não adotam as normas e os modelos para melhoria de processos apontam para questões associadas ao alto custo e à burocracia relacionada à grande quantidade de recursos demandados pela execução dos processos (Staples et al., 2007; Coleman e O'connor, 2008).

Outros fatores apontam para questões relacionadas a atitudes dos indivíduos, por exemplo, falta de motivação e resistência a mudanças pelos membros das organizações e falta de apoio e comprometimento da alta direção na iniciativa de melhoria (Baddoo, 2001; Niazi et al., 2006). Alguns estudos, também, indicam que diferenças culturais entre as organizações de software podem provocar variações nos relacionamentos e nas dependências entre esses fatores (El-Emam et al., 2001).

Constata-se, então, que a implementação de melhorias em processos é um fenômeno sócio-cultural. Para obter um entendimento preciso da prática de melhoria de processos é fundamental ir além do estudo de fatores tecnológicos (Dyba, 2000; ElEmam et al., 2001; Coleman e O'connor, 2007). No entanto, o estudo de questões nãotecnológicas, como o comportamento social humano, tem recebido pouca atenção pelos pesquisadores da área (Bertelsen, 1997; Seaman, 1999; Coleman e O'connor, 2007; Adolph et al., 2008).

Este trabalho apresenta uma investigação conduzida por meio da aplicação de métodos qualitativos e quantitativos de análise de dados, visando construir um framework teórico que ajude a explicar o processo social do comportamento humano que rege a implementação de melhorias em processos de software. O framework teórico é constituído de conceitos e relacionamentos de influência, fundamentados em um conjunto de proposições (hipóteses), representando a visão e a perspectiva de implementadores de melhorias em processos de software.

A seção 2 apresenta alguns estudos experimentais sobre os fatores críticos de sucesso em iniciativas de melhoria de processos de software. As seções 3 e 4 apresentam, respectivamente, a metodologia e a estrutura geral da pesquisa. A seção 5 apresenta os principais resultados obtidos na investigação conduzida no contexto deste trabalho. A seção 6 apresenta a conclusão e os próximos passos da investigação.

\section{Estudos Experimentais sobre os Fatores Críticos de Sucesso em Iniciativas de Melhoria de Processos de Software}

Wilson et al. (2001) desenvolveram um framework para avaliação do sucesso de iniciativas de melhorias em processos de software e validaram essa abordagem em um estudo envolvendo desenvolvedores, supervisores/líderes de equipe, alta gerência e coordenadores da melhoria de sete organizações do Reino Unido. Segundo os autores, os resultados permitiram confirmar a existência de questões críticas identificadas em outros estudos, como a importância do comprometimento da alta gerência, bem como identificaram novas questões, por exemplo a definição dos processos para serem implementados nos estágios iniciais da iniciativa de melhoria.

Baddoo e Hall (2002a) apresentaram os resultados de um estudo sobre os motivadores de melhorias de processos de software, envolvendo quase 200 engenheiros de software de 13 organizações do Reino Unido. Os motivadores identificados, nesse estudo, foram analisados em um estudo subseqüente, reportado em (Baddoo e Hall, 2002b). O principal objetivo deste novo estudo foi compreender o relacionamento entre 
os motivadores dos envolvidos em iniciativas de melhoria por meio da demonstração de como esses motivadores co-ocorrem uns com os outros. Essa co-ocorrência foi representada graficamente, utilizando a técnica de análise de dados denominada "Escalamento Multidimensional" (MDS, do inglês Multi-Dimensional Scaling). Os resultados desse estudo sugerem que existem relacionamentos significativos entre os motivadores, segundo cada grupo envolvido nas iniciativas de melhoria. Os autores identificaram um forte relacionamento entre os motivadores "compulsão" e "padronização". Segundo os autores, quando os desenvolvedores indicam que estão motivados por práticas compulsórias de melhoria de processos de software, eles também estão sugerindo que a padronização é um motivador.

Baddoo e Hall (2003) relataram um outro estudo com foco nos desmotivadores para implementação de melhorias em processos de software. Neste estudo, foi observado que os desmotivadores para implementação de melhoria de processos de software representam a ausência dos motivadores identificados em um estudo anterior, apresentado em (Baddoo e Hall, 2002b), sugerindo que alguns dos motivadores e desmotivadores podem ser interpretados como sendo parte do mesmo fator crítico de sucesso. Os fatores identificados pelos autores como desmotivadores para a melhoria de processo de software foram: (i) resistência a mudanças, (ii) falta de evidências da melhoria de processos, (iii) imposição da melhoria em processos de software, (iv) restrições de recursos e (v) pressões comerciais.

Rainer e Hall (2002) relataram os resultados de um estudo conduzido com o propósito de investigar os fatores críticos de sucesso que têm maior impacto ou nenhum impacto na melhoria de processos de software sob o ponto de vista de gerentes de melhoria de processos de software de empresas do Reino Unido e multinacionais. Foi encontrado, nesse estudo, um total de quatro fatores que os envolvidos nas iniciativas de melhoria, no geral, consideraram exercer maior influência no sucesso das iniciativas: (i) revisões, (ii) procedimentos e padrões, (iii) treinamento e mentoring e (iv) equipe experiente. $\mathrm{O}$ estudo encontrou também outros quatro fatores que as organizações mais maduras consideraram exercer maior impacto no sucesso de iniciativas de melhoria: (i) liderança interna, (ii) inspeções, (iii) apoio executivo e (iv) posse do processo interno. A partir desses oito fatores, quatro temas mais abrangentes foram identificados: (i) pessoas, (ii) processo, (iii) habilidades e (iv) liderança.

Rainer e Hall (2003) relataram os resultados de um outro estudo exploratório para obter maior conhecimento sobre os fatores que engenheiros de software acreditam afetar a melhoria de processos de software. Esse estudo foi baseado em estudos anteriores realizados pelos autores: (i) um survey (Rainer e Hall, 2002) e (ii) estudos de caso de organizações específicas (Hall et al., 2000 -a; Hall et al., 2000 -b; Rainer e Hall, 2001). Neste novo estudo, os autores analisaram, quantitativamente e qualitativamente, as palavras usadas pelos entrevistados nos grupos dos estudos de caso para obter um maior entendimento sobre os fatores que esses profissionais acreditaram afetar a melhoria dos processos de software. Os autores comparam essa análise com os resultados do survey.

Niazi et al. (2005) apresentaram um estudo sobre os fatores críticos de sucesso na implementação de melhorias em processos de software que envolveu a realização de entrevistas com 23 implementadores australianos e a revisão de 50 artigos da literatura sobre fatores críticos de sucesso. Os autores do estudo apontaram que as entrevistas 
sobre os fatores críticos de sucesso são uma oportunidade única para apoiar gerentes na obtenção de um melhor entendimento sobre suas necessidades de informação.

\section{Metodologia de Pesquisa}

Considerando as principais dificuldades e os problemas na adoção de modelos e normas para melhoria de processos, bem como as limitações dos estudos conduzidos na área sobre fatores críticos de sucesso em iniciativas de melhoria, foi identificada a necessidade de construir uma Teoria de Implementação de Melhorias de Processos de Software que pudesse contribuir para explicar as questões críticas relacionadas ao processo social do comportamento humano e que podem influenciar o sucesso de iniciativas de melhoria.

Sendo a Teoria de Implementação de Melhorias de Processos de Software uma teoria substantiva sobre um fenômeno social da área de melhoria de processos, métodos qualitativos são considerados adequados para guiar a sua construção (Seaman, 1999; Coleman e O'connor, 2007). Decidiu-se utilizar o método Grounded Theory, segundo a linha straussiana, para guiar a condução da investigação, neste trabalho, pois esse método estabelece um conjunto de procedimentos para condução de pesquisa qualitativa em áreas pouco exploradas, bem como é um método com efetividade comprovada para apoiar a coleta e análise de experiências de profissionais da área de software, visando a construção de teorias substantivas sobre o comportamento humano (Orlikowski, 1993; Coleman e O'connor, 2007; Crabtree et al., 2009).

A metodologia adotada procurou seguir os principais conceitos do método Grounded Theory: princípio de emergência, comparação constante, amostragem teórica e procedimentos de codificação. O princípio de emergência define que, tanto o produto final da pesquisa, quanto a definição do processo de pesquisa, devem ser emergentes, ou seja, devem ser desenvolvidos ao longo do processo de pesquisa (Strauss e Corbin, 1998; Matavire e Brown, 2008). A técnica principal do método Grounded Theory para descobrimento de teorias é a comparação constante. Essa técnica é utilizada para (i) determinar a exatidão dos dados, (ii) estabelecer os limites de generalização empírica, (iii) especificar um conceito, (iv) gerar a teoria e (v) verificar a teoria (Glaser e Strauss, 1967; Matavire e Brown, 2008). A amostragem teórica na Grounded Theory é direcionada pelas categorias e proposições emergentes, isto é, a decisão sobre as amostras de dados a serem coletadas é um processo contínuo que não pode ser determinado a priori (Adolph et al., 2008). Os tipos de procedimentos de codificação propostos pelo método Grounded Theory são: codificação aberta, codificação axial e codificação seletiva. Codificação aberta é o processo analítico a partir do qual conceitos e categorias são identificados e suas propriedades e dimensões são descobertas nos dados (Strauss e Corbin, 1998). Codificação axial é definida como o processo de relacionamento entre as categorias e suas respectivas subcategorias. Codificação seletiva envolve a identificação de uma categoria central a partir da qual todas as demais categorias estão relacionadas. A categoria central deve representar a essência do processo social que ocorre entre os envolvidos, podendo ser uma categoria existente ou uma nova categoria deve ser criada (Strauss e Corbin, 1998). A descrição completa da metodologia é apresentada em (Montoni e Rocha, 2010a). 


\section{Estrutura Geral da Pesquisa}

Procurou-se aplicar a metodologia de pesquisa de forma a evitar que idéias preconcebidas sobre o fenômeno estudado, exercessem algum tipo de influência no resultado da investigação. Para tanto, foram definidas as seguintes questões de pesquisa de forma aberta: (i) QP1 - Quais são os fatores capazes de influenciar o sucesso de iniciativas de melhoria? e (ii) QP2 - Como os implementadores de melhoria tratam os fatores capazes de influenciar o sucesso de iniciativas de melhoria?

Para investigar essas questões, dividiu-se a pesquisa em duas fases. A primeira fase envolveu a realização de um survey com 25 consultores e membros de organizações de software para identificar fatores críticos de sucesso, bem como uma revisão sistemática da literatura sobre fatores críticos de sucesso. Segundo o princípio de emergência do método Grounded Theory, o resultado da primeira fase da pesquisa foi utilizado para guiar a fase seguinte de forma a focar a investigação apenas nos fatores mais críticos, capazes de influenciar o sucesso de iniciativas de melhoria.

$\mathrm{Na}$ segunda fase da pesquisa, foram realizados um survey e entrevistas com implementadores de uma organização específica de consultoria em melhoria de processo. Os participantes desses estudos foram selecionados segundo o princípio de amostragem teórica do método Grounded Theory, o qual estabelece que as fontes de dados devem ser escolhidas de acordo com a capacidade em explorar os conceitos do problema ou fenômeno de interesse. Portanto, a organização selecionada para esta etapa da investigação foi a COPPE/UFRJ, devido à grande experiência da instituição na coordenação de iniciativas bem sucedidas.

Como resultado dos estudos realizados, foi construído um framework teórico, constituído de um conjunto de proposições (hipóteses) e um conjunto de categorias (conceitos) que tentam explicar as questões críticas relacionadas ao processo social do comportamento humano e que podem influenciar o sucesso de iniciativas de melhoria.

$\mathrm{Na}$ etapa final da investigação, foram conduzidos estudos específicos para avaliar o framework teórico construído. Esses estudos foram os seguintes: (i) uma avaliação das proposições (hipóteses) que compõem o framework teórico por meio de um survey com coordenadores de diferentes instituições implementadoras do modelo MPS; e (ii) uma verificação da existência das categorias do framework teórico com base na análise de relatórios produzidos por diferentes instituições implementadoras do modelo MPS, contendo descrições de melhores práticas e lições aprendidas na implementação de melhorias em processos de software. Para reduzir o viés na avaliação do framework teórico, foi realizada, ao final da investigação, uma auditoria da verificação por um especialista em melhoria de processo da COPPE/UFRJ.

\section{Resultados Obtidos na Investigação}

\subsection{Resultados da Primeira Fase da Investigação}

$\mathrm{Na}$ primeira fase, a investigação teve caráter exploratório. A primeira fase da pesquisa envolveu a realização de um survey com implementadores de melhorias em processos e membros de diferentes organizações de software, e um estudo baseado em revisão sistemática da literatura sobre fatores críticos de sucesso em iniciativas de melhoria. Técnicas de análise estatística de dados foram aplicadas, nesta primeira fase da 
pesquisa, visando identificar grupos de fatores com relacionamento estatisticamente significativo. A metodologia aplicada na primeira fase da investigação é detalhada em (Montoni e Rocha, 2007).

A coleta dos dados no survey foi realizada por meio da aplicação de dois tipos de questionários. Um questionário foi submetido a implementadores de melhoria em processos de software e o outro a membros de grupos de processo de organizações de software. No total, foram retornados no survey, 25 questionários contendo descrições gerais sobre fatores que influenciam iniciativas de melhoria. Desse conjunto, 14 questionários eram de implementadores de 3 diferentes organizações de consultoria e 11 questionários de profissionais representando 4 diferentes organizações de software. Apesar da quantidade total de questionários não ser muito grande, considerou-se que a diversidade das experiências dos indivíduos era adequada para realizar uma exploração inicial dos conceitos do fenômeno de estudo. Os questionários foram inicialmente analisados pela aplicação dos procedimentos de codificação do método Grounded Theory. Cada passagem de texto dos questionários foi analisada linha a linha procurando identificar similaridades e diferenças entre os dados. A partir dessa análise, foi possível classificar os dados de acordo com o tipo de influência sobre um fator crítico de sucesso em iniciativas de melhoria.

O estudo baseado na revisão da literatura foi conduzido por meio da execução de um protocolo de pesquisa testado e avaliado visando garantir sua adequação para o contexto da pesquisa. No total, foram selecionados no estudo 15 trabalhos que descreviam os resultados de 13 estudos envolvendo profissionais de software que participaram de iniciativas de melhoria conduzidas em organizações de diferentes países (Reino Unido, Noruega, Malásia, Brasil, Índia e Bangladesh). Os trabalhos foram revistos com base no protocolo de pesquisa. Essa revisão possibilitou a extração de dados necessários para a realização de análises quantitativas e qualitativas sobre os fatores críticos de sucesso em iniciativas de melhoria. Em ambos os estudos, os fatores críticos de sucesso foram identificados por meio da categorização dos achados.

Um gráfico de Pareto (Figura 1) foi elaborado com base na contabilização realizada das ocorrências de propriedades de fatores críticos de sucesso no survey e na revisão sistemática da literatura. Decidiu-se apresentar apenas as ocorrências dessa categoria, pois é a categoria que apresenta a maior riqueza de detalhes sobre fatores críticos de sucesso.

Analisando os resultados (Figura 1) das 25 propriedades investigadas, 5 delas compreendem mais de $50 \%$ das ocorrências nos dois estudos, podendo, então, serem consideradas as mais críticas para alcançar sucesso na condução de iniciativas de melhoria. Essas propriedades são: "[P13] Competências em engenharia de software dos membros da organização (conhecimento, experiências e habilidades)", "[P11] Apoio efetivo da alta gerência", "[P06] Existir gerência do projeto de implementação da melhoria dos processos", "[P09] Adequação dos processos/procedimentos definidos" e "[P16] Conscientização dos membros da organização quanto aos benefícios obtidos com a implantação dos processos". 


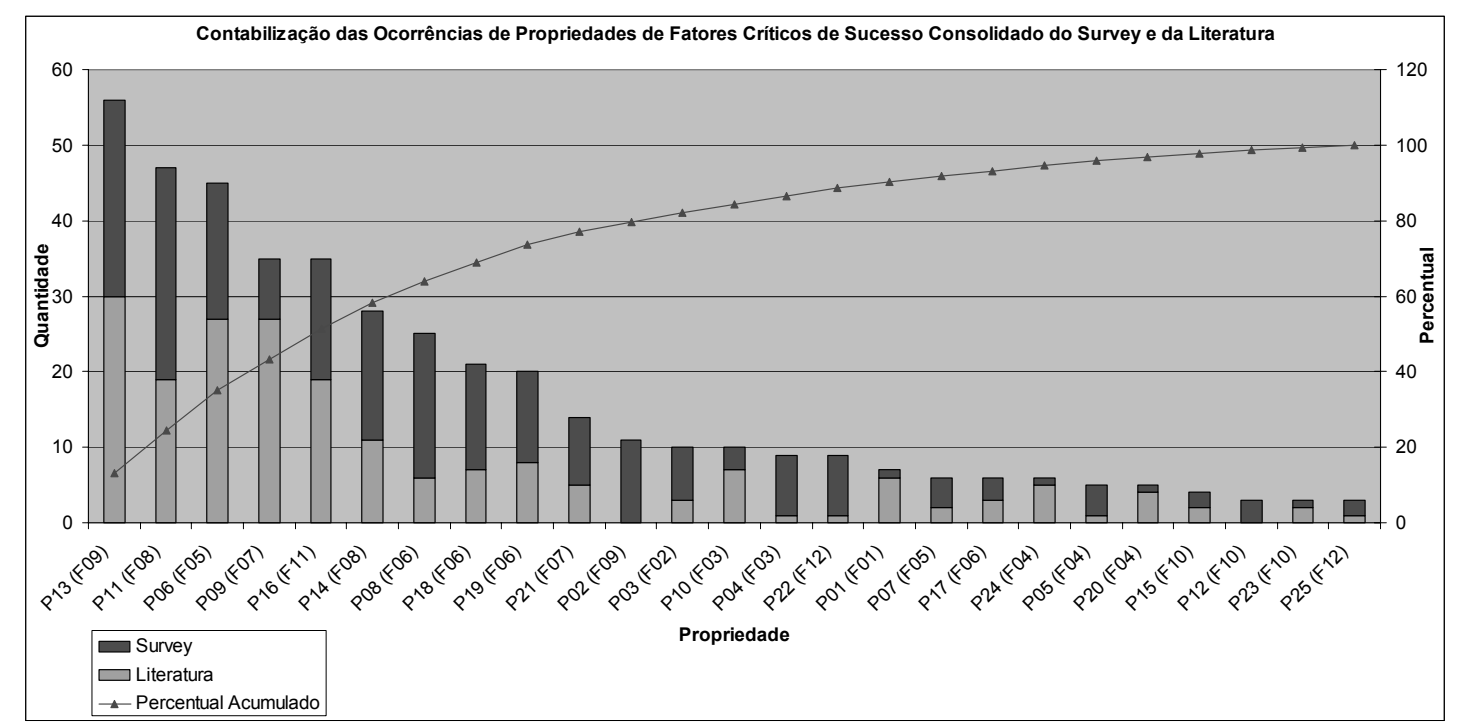

\section{Legenda:}

[P01] Existência de política de reconhecimento à colaboração na melhoria dos processos

[P02] Freqüência adequada de apoio de consultoria especializada

[P03] Facilidade de aceitação de mudanças

[P04] Inexistência de conflitos de interesses na implementação de processos

[P05] Estrutura da organização adequada

[P06] Existir gerência do projeto de implementação da melhoria dos processos

[P07] Balanceamento entre as melhorias impostas pela alta direção, as necessidades do modelo adotado e as melhorias propostas pelos membros da organização

[P08] Adequação das ferramentas de apoio

[P09] Adequação dos processos/procedimentos definidos

[P10] Alinhamento da definição dos processos com

objetivos estratégicos da organização

[P11] Apoio efetivo da alta gerência

[P12] Competências da consultoria especializada

(conhecimento, experiências e habilidades)

[P13] Competências em engenharia de software dos

membros da organização (conhecimento, experiências e

habilidades)
[P14] Comprometimento e envolvimento dos membros da organização
[P15] Confiança dos membros da organização na consultoria especializada
[P16] Conscientização dos membros da organização quanto aos benefícios obtidos com a implantação dos processos
[P17] Disponibilidade de recursos de software e hardware de apoio à execução dos processos
[P18] Disponibilidade de recursos financeiros da organização para atividades de melhoria de processo [P19] Disponibilidade de tempo dos membros da organização para atividades de melhoria de processo [P20] Estabilidade interna na organização
[P21] Completa institucionalização das melhorias implementadas nos projetos
[P22] Motivação dos membros da organização
[P23] Relacionamento dos membros da organização com a consultoria especializada
[P24] Rotatividade de pessoal da organização
[P25] Satisfação dos membros da organização

\section{Figura 1. Gráfico de Pareto da contabilização das ocorrências de propriedades de fatores críticos de sucesso no survey e na revisão sistemática da literatura}

Esse resultado indica que uma iniciativa de melhoria, para ter sucesso, deve garantir competências adequadas das pessoas responsáveis por definir, implementar e adotar as melhorias nos processos dos projetos de software. Além disso, é fundamental garantir o apoio efetivo da alta gerência. O resultado também indica a importância da conscientização dos envolvidos, quanto aos benefícios esperados com a implementação de melhorias em processos de software. Outros aspectos críticos de caráter mais técnico estão relacionados à capacidade organizacional em conduzir as iniciativas de melhoria como projetos reais, bem como à adequação dos processos e procedimentos definidos.

Um segundo grupo também crítico para o sucesso de iniciativas de melhoria é composto das seguintes propriedades: "[P14] Comprometimento e envolvimento dos membros da organização", "[P08] Adequação das ferramentas de apoio", "[P18] 
Disponibilidade de recursos financeiros da organização para atividades de melhoria de processo" e "[P19] Disponibilidade de tempo dos membros da organização para atividades de melhoria de processo".

Esse segundo grupo indica que é preciso ter um comprometimento e envolvimento adequado dos membros da organização. Além disso, indica que é importante realizar um planejamento adequado da alocação de recursos para a iniciativa de melhoria. Esses recursos abrangem tanto recursos financeiros e ferramentas de apoio aos processos, quanto alocação de tempo das pessoas responsáveis pela implementação das melhorias nos processos.

No entanto, nota-se no gráfico da Figura 1 que não foram identificadas ocorrências de algumas propriedades nos estudos da literatura. Essas propriedades foram: "[P02] Freqüência adequada de apoio de consultoria especializada" e "[P12] Competências da consultoria especializada (conhecimento, experiências e habilidades)". Essas categorias foram criadas a partir do survey, envolvendo implementadores de organizações de consultoria. No entanto, os trabalhos da literatura envolviam exclusivamente profissionais de organizações de software. Devido a isso, pode ser que esses profissionais não reconheçam como relevante ter uma freqüência adequada de apoio de consultoria, nem competências adequadas dos consultores para ter sucesso.

A análise realizada teve o intuito de facilitar a priorização no tratamento das questões mais críticas para alcançar sucesso na condução de iniciativas de melhoria. No entanto, apesar das demais propriedades e fatores não terem tido grande número de ocorrências nos estudos, não se pode concluir que esses fatores não são críticos. É possível que, em determinados contextos, esses fatores sejam capazes de exercer influência direta ou indireta no sucesso de iniciativas de melhoria.

Além disso, o resultado da análise dos fatores com relacionamento estatisticamente significativo apontou indícios da inter-dependência entre alguns dos fatores com baixa ocorrência na revisão da literatura e os demais fatores. Por exemplo, as propriedades com menor ocorrência nos dois estudos ("[P23] Relacionamento dos membros da organização com a consultoria especializada" e "[P25] Satisfação dos membros da organização"), apresentaram ter um relacionamento estatisticamente significativo com a propriedade de maior ocorrência ("[P13] Competências em engenharia de software dos membros da organização (conhecimento, experiências e habilidades)"). Portanto, conclui-se que mesmo as propriedades e fatores com baixa ocorrência nos dados devem ser considerados, tanto em investigações futuras, quanto no gerenciamento de iniciativas de melhoria.

\subsection{Resultados da Segunda Fase da Investigação}

O objetivo desta segunda fase do estudo foi investigar como implementadores de melhoria tratam os fatores capazes de influenciar o sucesso de iniciativas de melhoria. Esta fase do estudo foi conduzida em cinco etapas: (i) Condução de um survey com os implementadores da COPPE/UFRJ, (ii) Realização de entrevistas semi-estruturadas com os implementadores experientes da COPPE/UFRJ, (iii) Condução de um survey com os coordenadores das instituições implementadoras do modelo MPS, (iv) Verificação da teoria construída nesta fase do estudo e (v) Auditoria da verificação dessa teoria. A metodologia aplicada na segunda fase da investigação é detalhada em (Montoni e Rocha, 2010b). 
Segundo o princípio de emergência do método Grounded Theory, à medida que progride um estudo qualitativo, deve-se explorar tópicos mais específicos, definidos de acordo com os resultados obtidos nos estágios anteriores da pesquisa. Além disso, devese garantir, também, uma amostragem teórica adequada por meio da seleção de fontes de dados com potencial para explorar os tópicos de interesse. Alinhado a isso, decidiuse iniciar a investigação, nesta segunda fase do estudo, por meio da condução de um survey, visando determinar o grau de influência dos fatores críticos de sucesso, identificados na primeira fase do estudo. A identificação do grau de influência dos fatores pode ajudar a entender melhor as questões críticas em iniciativas de melhoria, possibilitando a definição de estratégias mais eficazes de implementação. A instituição selecionada para participar do survey foi a COPPE/UFRJ, devido ao grande número de experiências passadas com iniciativas de melhoria de processos de software de sucesso, coordenadas por essa instituição.

A etapa seguinte, nesta segunda fase do estudo, envolveu a realização de entrevistas semi-estruturadas com os implementadores mais experientes da COPPE/UFRJ. Essas entrevistas foram guiadas por um questionário, desenvolvido especificamente para explorar questões relacionadas ao contexto e às formas de tratamento dos fatores críticos de sucesso.

Os dados das entrevistas realizadas foram analisados por meio da aplicação dos procedimentos de codificação do método Grounded Theory. Como resultado da análise, foi estendido o conjunto de categorias de fatores críticos de sucesso, bem como foram definidas as inter-dependências entre essas categorias. Um conjunto de proposições (hipóteses) também foi elaborado e avaliado pelos entrevistados, visando fornecer descrições concisas dos relacionamentos entre as categorias. Essas proposições e categorias formam o framework teórico, construído com o propósito de explicar o processo social que rege a implementação de melhorias em processos de software. Um meta-modelo foi proposto para apoiar a identificação dos conceitos (categorias) do framework teórico construído.

Em seguida, foi realizada uma avaliação do framework teórico por meio de um survey com coordenadores de instituições implementadoras do modelo MPS. O objetivo do survey foi verificar o quanto a teoria desenvolvida, representa a visão e perspectiva dos implementadores de melhoria de diferentes instituições de consultoria. Como resultado dessa avaliação, foi possível distinguir entre as proposições que representam, de forma geral ou específica, a perspectiva das instituições implementadoras do modelo MPS.

As etapas finais, nesta segunda fase do estudo, tiveram como objetivo complementar a avaliação do framework teórico por meio da verificação da existência dos conceitos da teoria (categorias) em um conjunto específico de fontes de dados, contendo descrições sobre melhores práticas e lições aprendidas na implementação de melhorias em processos. Foram utilizados como fontes de dados, todos os relatórios semestrais elaborados pelos coordenadores das instituições implementadoras do modelo MPS, credenciadas pela SOFTEX. O resultado dessa verificação foi a identificação de passagens de textos, extraídas dos relatórios, que evidenciam a existência dos conceitos da teoria desenvolvida no estudo. Visando reduzir o viés dessa verificação, foi feita uma auditoria da comprovação da existência dos conceitos por um especialista em melhoria de processo. 
Como resultado do estudo, foram identificados dois conjuntos de categorias centrais denominadas de "Contexto Institucional para Implementação de Melhorias em Processos de Software" e "Comportamento Estratégico na Implementação de Melhorias em Processos de Software", representando, respectivamente, as características de contexto que podem influenciar o sucesso de uma iniciativa de melhoria, e as ações estratégicas realizadas por atores da implementação de melhorias em processos, capazes de reforçar ou modificar tais influências.

Para facilitar a análise das relações de influência entre os conceitos, foram extraídos grupos de propriedades inter-relacionadas. O conjunto de propriedades de conceitos inter-relacionadas foi denominado de "Componente". De maneira diferente da primeira fase do estudo, na qual foram aplicadas técnicas de análise estatística de dados para extração de fatores inter-dependentes, nesta segunda fase do estudo, havia mais conhecimento sobre os fatores, possibilitando que a identificação dos componentes fosse realizada de forma qualitativa por meio da análise das proposições da teoria, e que definem os relacionamentos entre as propriedades.

Foram identificados seis componentes de relacionamento entre as propriedades, denominados de: (i) "Aceitação e Comprometimento", (ii) "Apoio e Relacionamento", (iii) "Melhorias nos Processos de Software", (iv) "Capacitação e Gerência", (v) "Recursos", e (vi) "Retorno do Programa de Melhoria de Processos". A Figura 2 apresenta um exemplo do esquema teórico construído com base nas relações entre as propriedades do componente "Aceitação e Comprometimento".

Conforme observado na Figura 2, duas forças principais atuam, influenciando a facilidade de aceitação de mudanças pelos membros da organização. A primeira delas reside nas próprias características dos membros da organização que são a motivação e conscientização dos benefícios com as melhorias, além das competências e facilidade de aprendizagem. A segunda força diz respeito às atitudes da alta gerência que são a influência da alta gerência na organização e a efetividade do seu apoio na implementação das melhorias nos processos. O comprometimento e envolvimento dos membros da organização estão diretamente relacionados à motivação das pessoas para a melhoria. A perspectiva de avaliação formal também pode exercer algum tipo de influência no comprometimento e envolvimento das pessoas na iniciativa de melhoria. No contexto organizacional, foi percebido que a capacidade do programa de melhoria tornar visível o retorno do investimento nas melhorias nos processos pode influenciar, indiretamente, a aceitação e comprometimento dos membros da organização, por exemplo, ajudando a aumentar a efetividade do apoio da alta gerência, bem como a motivar e conscientizar os membros da organização para a melhoria. A disponibilidade de recursos financeiros pode ajudar a aumentar o apoio da alta gerência e, conseqüentemente, facilitar a aceitação de mudanças. 


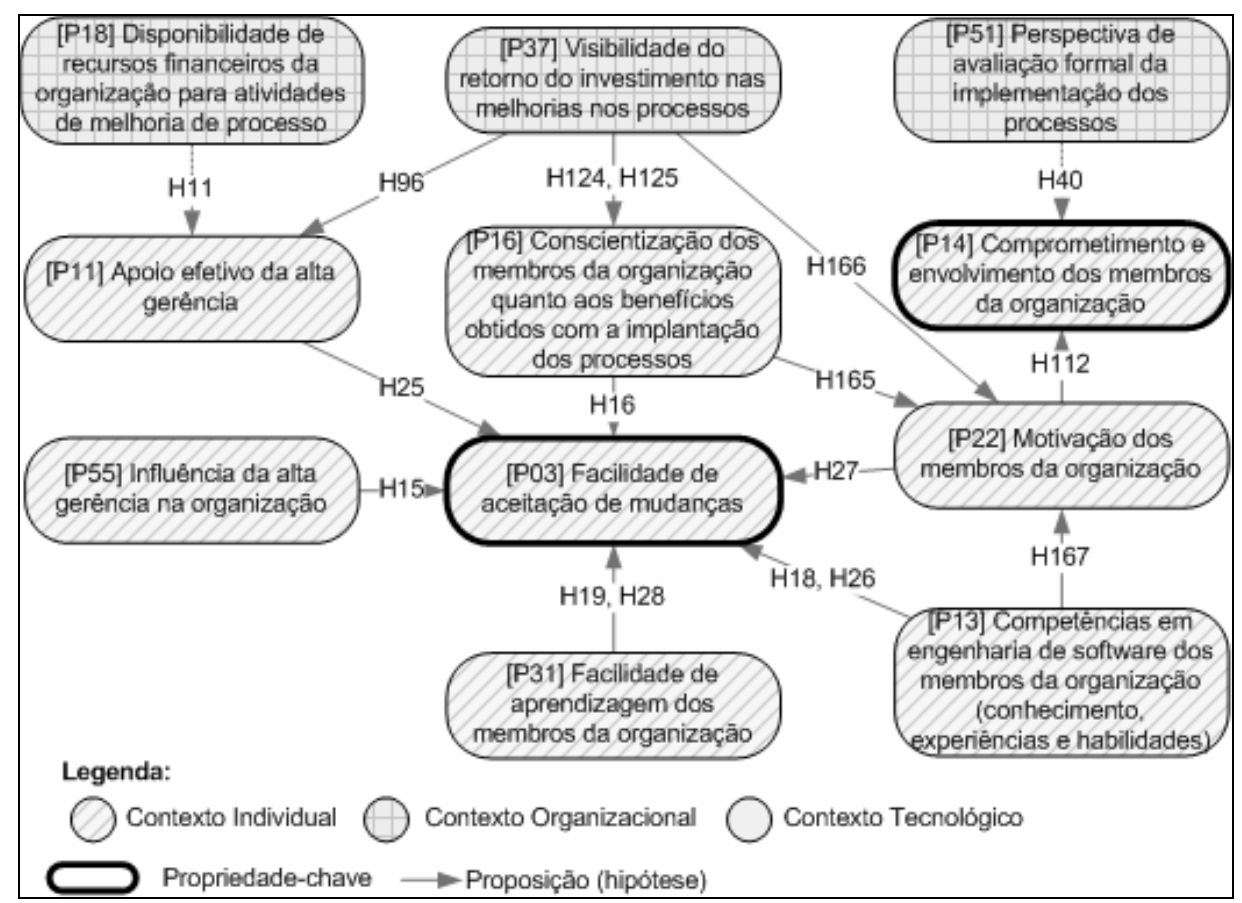

Figura 2. Representação das relações entre as propriedades do componente "Aceitação e Comprometimento"

A Tabela 1 apresenta exemplos das proposições que fundamentam as relações apresentadas na Figura 2.

Tabela 1. Exemplos de proposições (hipóteses)

\begin{tabular}{|l|l|}
\hline \multicolumn{2}{|c|}{ Hipótese } \\
\hline H26 & $\begin{array}{l}\text { Quando os profissionais da empresa são altamente capacitados é mais provável ter-se facilidade } \\
\text { de aceitação de mudanças. Nota de análise: No contexto da análise, pode ser inferido que a } \\
\text { capacitação está relacionada às competências em engenharia de software. }\end{array}$ \\
\hline H27 & $\begin{array}{l}\text { Quando a equipe da empresa é motivada para melhoria de processos, é mais provável ter-se } \\
\text { facilidade de aceitação de mudanças. }\end{array}$ \\
\hline H112 & $\begin{array}{l}\text { Quando os membros da organizção não demonstram interesse ou motivação por melhorias nos } \\
\text { processos, é provável ter-se problemas por falta de comprometimento e envolvimento dos } \\
\text { membros da organização com o projeto de melhoria. }\end{array}$ \\
\hline
\end{tabular}

Análises e proposições (hipóteses) como as apresentadas acima foram identificadas para cada um dos seis componentes de fatores críticos de sucesso resultantes da segunda fase da investigação, mas por limitação de espaço não foram apresentadas neste trabalho.

Esquemas teóricos e análises como as apresentadas acima também foram elaborados para as cinco categorias de subprocessos que agregam os conceitos que representam o comportamento ou o processo de mudança organizacional, do ponto de vista da instituição de consultoria, e que descrevem a interação entre os atores da iniciativa de melhoria e o contexto institucional no qual as melhorias são implementadas (Iniciando o projeto de consultoria em melhoria de processos, Planejando o projeto de consultoria em melhoria de processos, Implantando melhorias nos processos de software, Acompanhando e controlando a implantação das melhorias nos processos, e Gerenciando os recursos humanos da consultoria especializada em melhoria de processos). 
Os últimos passos da segunda fase da investigação envolveram a avaliação do framework teórico por meio da condução de dois estudos. Primeiro, foi realizada uma avaliação do framework teórico por meio de um survey com coordenadores de instituições implementadoras do modelo MPS. O objetivo do survey foi verificar o quanto a teoria desenvolvida, representa a visão e perspectiva dos implementadores de melhoria de diferentes instituições de consultoria. Como resultado dessa avaliação, foi possível distinguir entre as proposições que representam, de forma geral ou específica, a perspectiva das instituições implementadoras do modelo MPS. Em seguida, foi realizada uma avaliação do framework teórico por meio da verificação da existência dos conceitos da teoria (categorias) em um conjunto específico de fontes de dados, contendo descrições sobre melhores práticas e lições aprendidas na implementação de melhorias em processos. Foram utilizados como fontes de dados, todos os relatórios semestrais elaborados pelos coordenadores das instituições implementadoras do modelo MPS, credenciadas pela SOFTEX. O resultado dessa verificação foi a identificação de passagens de textos, extraídas dos relatórios, que evidenciam a existência dos conceitos da teoria desenvolvida no estudo. Visando reduzir o viés dessa verificação, foi feita uma auditoria por um especialista em melhoria de processo. As Figuras 3 e 4 apresentam, respectivamente, os resultados da verificação das proposições e das categorias que compõem o framework teórico construído nesta investigação.
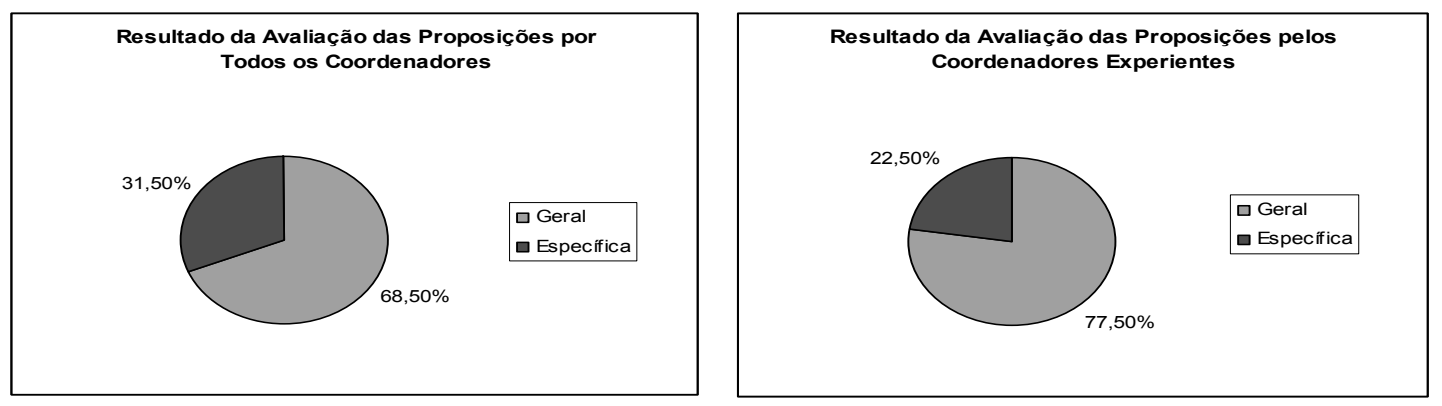

Figura 3. Resultado da avaliação das proposições pelos coordenadores de instituições implementadoras do modelo MPS

Conforme observado, na Figura 3, os resultados das avaliações dos coordenadores fornecem indícios de que as proposições representam em determinado grau a visão e a perspectiva dos coordenadores. Os coordenadores concordaram, em média, com mais de $68 \%$ das proposições. Esse número é ainda maior, considerando apenas os coordenadores experientes. Estes últimos concordaram, em média, com mais de $77 \%$ das proposições.
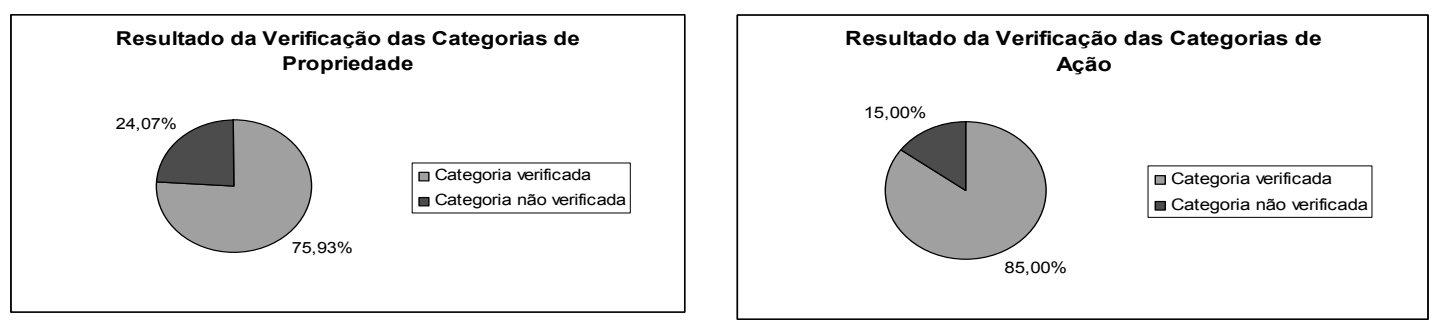

Figura 4. Resultado da verificação das categorias da teoria 
O resultado apresentado na Figura 4 mostra que, uma grande parte das categorias que compõem a teoria (mais de $75 \%$ das propriedades e $85 \%$ das ações) foram verificadas nos relatórios das instituições implementadoras do modelo MPS. Esse resultado é significativo, pois a verificação foi realizada utilizando fontes de dados originadas em contextos diferentes do contexto no qual este estudo foi conduzido. Além disso, a realização, com sucesso, da auditoria da verificação, ajuda a aumentar a confiabilidade dos resultados obtidos.

\section{Conclusão}

Este trabalho apresentou uma investigação conduzida por meio da aplicação de métodos qualitativos e quantitativos de análise de dados, visando construir um framework teórico que ajude a explicar o processo social do comportamento humano que rege a implementação de melhorias em processos de software. Os estudos conduzidos durante a execução da investigação foram apresentados, bem como os principais resultados obtidos. Os resultados desta pesquisa confirmaram que a implementação de melhorias em processos de software é de fato um processo social. Essa constatação foi comprovada pela identificação de questões críticas associadas ao relacionamento entre os consultores da instituição implementadora e os membros da organização alvo da implementação das melhorias. Um fator que influência esse relacionamento é a confiança dos membros da organização na consultoria especializada.

Como principal contribuição deste trabalho, destaca-se a construção de um framework teórico que ajuda a explicar o processo social do comportamento humano que rege a implementação de melhorias em processos no contexto do setor de software do Brasil. Além disso, também constitui uma contribuição, a aplicabilidade do método Grounded Theory para investigar os fatores críticos de sucesso em iniciativas de melhoria de processos de software, bem como o meta-modelo desenvolvido para organizar os conceitos e relacionamentos do framework teórico.

Por fim, podem ser destacadas algumas limitações deste trabalho. O framework teórico, construído, descreve o contexto e processo de mudança organizacional que as organizações de software podem experimentar durante a implementação de melhorias em processos de software. No entanto, é importante notar que os relacionamentos e as dependências apresentadas não são determinísticos. Mudanças organizacionais provocadas pela implementação de melhorias nem sempre serão as intencionadas pelos envolvidos na iniciativa de melhoria. Outra limitação do trabalho é que o framework teórico foi desenvolvido com base na visão de consultores de implementação de melhorias em processos. Portanto, os resultados apresentados não tratam da perspectiva específica de outros profissionais envolvidos nas iniciativas de melhoria, como os membros da organização alvo da implementação.

Os próximos passos deste trabalho envolvem a realização de novas investigações com o propósito de replicar a pesquisa em diferentes contextos, bem como aprofundamento da avaliação do framework teórico.

\section{Referências}

Adolph, S., Hall, W., Kruchten, P., 2008, "A methodological leg to stand on: Lessons learned using grounded theory to study software development", pp. IBM 
Toronto Software Lab.; IBM Centers for Advanced Studies, CAS, Richmond Hill, ON, Canada.

Baddoo, N., 2001, Motivators and de-motivators in software process improvement: an empirical study, $\mathrm{PhD}$, University of Hertfordshire, UK.

Baddoo, N., Hall, T., 2002a, "Motivators of Software Process Improvement: An analysis of practitioners' views", Journal of Systems and Software, v. 62, n. 2, pp. 85-96.

Baddoo, N., Hall, T., 2002b, "Software process improvement motivators: An analysis using multidimensional scaling", Empirical Software Engineering, v. 7, n. 2, pp. 93-114.

Baddoo, N., Hall, T., 2003, "De-motivators for software process improvement: An analysis of practitioners' views", Journal of Systems and Software, v. 66, n. 1, pp. 23-33.

Bertelsen, O.W., 1997, "Toward a unified field of SE research and practice", IEEE Software, v. 14, n. 6, pp. 87-88.

Coleman, G., O'connor, R., 2007, "Using grounded theory to understand software process improvement: A study of Irish software product companies", Information and Software Technology, v. 49, n. 6, pp. 654-667.

Coleman, G., O'connor, R., 2008, "Investigating software process in practice: A grounded theory perspective", Journal of Systems and Software, v. 81, n. 5, pp. $772-784$.

Crabtree, C.A., Seaman, C.B., Norcio, A.F., 2009, "Exploring language in software process elicitation: A grounded theory approach", IEEE Computer Society.

Dyba, T., 2000, "An Instrument for measuring the key factors of success in software process improvement", Empirical Software Engineering, v. 5, n. 4, pp. 357-390.

El-Emam, K., Goldenson, D., Mccurley, J., et al., 2001, "Modelling the likelihood of software process improvement: An exploratory study", Empirical Software Engineering, v. 6, n. 3, pp. 207-229.

Glaser, B.G., Strauss, A., 1967, The Discovery of Grounded Theory: Strategies for Qualitative Research Chicago / Illinois, Aldine.

Hall, T., Baddoo, N., Wilson, D., 2000 -a, "Measurement in Software Process Improvement Programmes: An Empirical Study". In: Proceedings of the 10th International Workshop on New Approaches in Software Measurement, pp. 7382.

Hall, T., Wilson, D.N., Baddoo, N., 2000 -b, "Towards Implementing Successful Software Inspections ". In: Proceedings of the International Conference on software Methods and Tools (SMT'00).

Matavire, R., Brown, I., 2008, "Investigating the use of "Grounded Theory" in information systems research", $A C M$, Wilderness, South Africa.

Montoni, M., Rocha, A.R., 2007, "A Methodology for Identifying Critical Success Factors that Influence Software Process Improvement Initiatives: An 
Application in the Brazilian Software Industry", Lecture Notes in Computer Science (LNCS), LNCS 4764, EuroSPI - European Systems \& Software Process Improvement and Innovation (Setembro), pp. 175-186.

Montoni, M.A., Rocha, A.R., 2010a, "Aplicação de Grounded Theory para Investigar Iniciativas de Implementação de Melhorias em Processos de Software". In: IX Simpósio Brasileiro de Qualidade de Software, pp. 167-182, Belém, Junho.

Montoni, M.A., Rocha, A.R., 2010b, "Applying Grounded Theory to Understand Software Process Improvement Implementation". In: 7th International Conference on the Quality of Information and Communications Technology, pp. 25-34, Porto, Portugal, 29 de setembro a 02 de outubro.

Niazi, M., Wilson, D., Zowghi, D., 2005, "A maturity model for the implementation of software process improvement: An empirical study", Journal of Systems and Software, v. 74, n. 2 SPEC ISS, pp. 155-172.

Niazi, M., Wilson, D., Zowghi, D., 2006, "Critical success factors for software process improvement implementation: An empirical study", Software Process Improvement and Practice, v. 11, n. 2, pp. 193-211.

Orlikowski, W.J., 1993, "CASE Tools as Organizational Change: Investigating Incremental and Radical Changes in Systems Development", Management Information Systems Quarterly, v. 17, n. 3.

Rainer, A., Hall, T., 2001, "An analysis of some 'core studies' of software process improvement", Software Process: Improvement and Practice, v. 6, n. 4, pp. 169187.

Rainer, A., Hall, T., 2002, "Key success factors for implementing software process improvement: A maturity-based analysis", Journal of Systems and Software, v. 62, n. 2 , pp. $71-84$.

Rainer, A., Hall, T., 2003, "A quantitative and qualitative analysis of factors affecting software processes", Journal of Systems and Software, v. 66, n. 1, pp. 7-21.

Seaman, C.B., 1999, "Qualitative methods in empirical studies of software engineering", IEEE Transactions on Software Engineering, v. 25, n. 4, pp. 557572.

Sei, 2006, CMMI® for Development (CMMI-DEV), V1.2, CMU/SEI-2006-TR-008, Software Engineering Institute.

Softex, 2009, "MPS.BR - Melhoria de Processo do Software Brasileiro, Guia Geral (v. 2009)". In: http://www.softex.br/mpsbr/_guias/default.asp.

Staples, M., Niazi, M., Jeffery, R., et al., 2007, "An exploratory study of why organizations do not adopt CMMI", Journal of Systems and Software, v. 80, n. 6, pp. 883-895.

Strauss, A., Corbin, J.M., 1998, Basics of Qualitative Research: Techniques and Procedures for Developing Grounded Theory, 2nd ed., Sage Publications.

Wilson, D.N., Hall, T., Baddoo, N., 2001, "A framework for evaluation and prediction of software process improvement success", Journal of Systems and Software, v. 59, n. 2, pp. 135-142. 